\title{
The Sunburst Arc: Direct Lyman $\alpha$ escape observed in the brightest known lensed galaxy ${ }^{\star}$
}

\author{
T. E. Rivera-Thorsen ${ }^{1}$, H. Dahle ${ }^{1}$, M. Gronke ${ }^{1}$, M. Bayliss ${ }^{2}$, J. R. Rigby ${ }^{3}$, R. Simcoe ${ }^{2}$, R. Bordoloi ${ }^{2, \star \star}$, \\ M. Turner ${ }^{2,4}$, and G. Furesz ${ }^{2}$ \\ 1 Institute of Theoretical Astrophysics, University of Oslo, Postboks 1029, 0315 Oslo, Norway \\ e-mail: eriveth@astro.uio.no \\ 2 MIT-Kavli Center for Astrophysics and Space Research, 77 Massachusetts Avenue, Cambridge, MA 02139, USA \\ 3 Observational Cosmology Lab, NASA Goddard Space Flight Center, 8800 Greenbelt Rd., Greenbelt, MD 20771, USA \\ ${ }^{4}$ Las Cumbres Observatory, 6740 Cortona Dr, Goleta, CA 93117, USA \\ Received 25 October 2017 / Accepted 19 November 2017
}

\begin{abstract}
We present rest-frame ultraviolet and optical spectroscopy of the brightest lensed galaxy yet discovered, at redshift $z=2.4$. The source reveals a characteristic triple-peaked Lyman $\alpha$ profile that has been predicted in various theoretical works, but to our knowledge has not been unambiguously observed previously. The feature is well fit by a superposition of two components: a double-peak profile emerging from substantial radiative transfer, and a narrow, central component resulting from directly escaping Lyman $\alpha$ photons, but it is poorly fit by either component alone. We demonstrate that the feature is unlikely to contain contamination from nearby sources, and that the central peak is unaffected by radiative transfer effects except for very slight absorption. The feature is detected at signal-to-noise ratios exceeding 80 per pixel at line center, and bears strong resemblance to synthetic profiles predicted by numerical models.
\end{abstract}

Key words. galaxies: individual: PSZ1-ARC G311.660218.4624 - galaxies: starburst - gravitational lensing: strong Galaxy: evolution - galaxies: high-redshift - galaxies: ISM

\section{Introduction}

Young star-forming galaxies are generally believed to be the most important source of the Lyman continuum (LyC) radiation that is responsible for the reionization of the early Universe (e.g., Bouwens et al. 2012; Faisst 2016). However, faint galaxies with high star formation rates tend to contain large amounts of neutral hydrogen, which is opaque to LyC at column densities $\log (N) \gtrsim 17.2$ (e.g., Osterbrock \& Ferland 2006). Exactly where in the galaxy this radiation originates and how it finds its way into the intergalactic medium remains one of the most important unsolved problems in astrophysics.

Lyman continuum radiation can be allowed to escape a galaxy mainly by two means. In one scenario, the source regions can be surrounded by a largely isotropic gas with a sufficiently low column density of neutral hydrogen $N_{\mathrm{HI}}$ to not completely attenuate the passing LyC radiation. This is usually referred to as the density-bounded medium. In a second scenario, the neutral medium is optically thick, but does not completely cover the ionizing sources. This scenario has been referred to as the picket fence model (e.g., Conselice et al. 2000; Heckman et al. 2011), the riddled ionization-bounded medium (Verhamme et al. 2015), or the ionization-bounded medium with holes (Zackrisson et al. 2013). The latter scenario can be subdivided into two cases: one quasi-isotropic, where the medium consists of clumps with a combined covering fraction below unity. This scenario will potentially have a large number of narrow direct lines of sight

\footnotetext{
* Based on observations obtained at the Magellan-I (Baade) Telescope at Las Campanas Observatory, Chile.

$\star \star$ Hubble Fellow.
}

(LOS) in random directions (Hansen \& Oh 2006; Duval et al. 2014; Gronke et al. 2016). In the other case, the medium is closer to an optically thick shell that is perforated in a few places by channels of very low optical depth (e.g., Zackrisson et al. 2013; Behrens et al. 2014). The clumpy and the perforated shell cases are not always well distinguished in the literature; we here reserve the term "picket fence model" for the clumpy scenario. While these three scenarios have similar observational signatures in LyC, they have different spectral signatures in Lyman $\alpha$, which is shaped by resonant interactions with the $\mathrm{HI}$ that also governs the escape of LyC, although in different ways (see, e.g., Dijkstra 2014, and references therein).

Figure 1 shows a cartoon depiction of these three scenarios and their emerging $\operatorname{Ly} \alpha$ spectral shapes. Blue arrows signify $\operatorname{Ly} \alpha$, and red arrows LyC radiation. The left panel shows the density-bounded model. Lyman $\alpha$, which has an interaction cross section $\sim 3$ orders of magnitude higher at line center, undergoes substantial radiative transfer before escaping as a broadened, double-peaked profile, while LyC escapes because the optical depth of the medium is low (Jaskot \& Oey 2013, 2014; Verhamme et al. 2015). Most local LyC leakers display Ly $\alpha$ line shapes similar to this (Verhamme et al. 2015), and are also in metal absorption more consistent with this scenario (Chisholm et al. 2017). The central panel shows the picket fence model, in which LyC can escape only through open sight lines between dense clumps, while Ly $\alpha$ can bounce off the boundaries of clumps and thus escape after a few scatterings and only weak radiative transfer effects. The resulting Ly $\alpha$ spectrum is a narrow single-peaked line whose intrinsic shape is nearly intact (e.g., Gronke et al. 2016). The right panel shows the perforated shell 


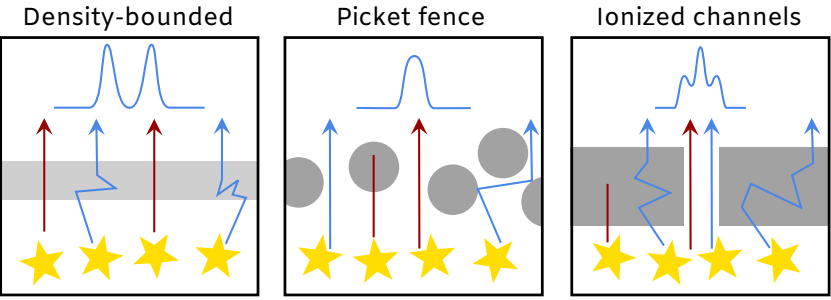

Fig. 1. Cartoons showing a fully covering $\lesssim 10^{17} \mathrm{~cm}^{-2} \mathrm{H} \mathrm{I}$ screen (left), the picket fence scenario of a clumpy medium with many ionized sight lines and weak radiative transfer effects in Ly $\alpha$ (center), and the scenario with a few ionized channels through a neutral medium that is optically thick in both $\operatorname{LyC}$ and $\operatorname{Ly} \alpha(r i g h t)$. Blue arrows show $\operatorname{Ly} \alpha$, and red arrows LyC.

model. Here, LyC and a central Ly $\alpha$ peak also escape directly through an open channel. In addition, Ly $\alpha$ photons are captured scattering in the denser neutral gas, which leads to substantial radiative transfer effects. The resulting Ly $\alpha$ line shape is a narrow central peak that escapes through the uncovered sight lines, which is superimposed on the characteristic double-peak profile that emerges from the optically thick gas (e.g., Duval et al. 2014; Behrens et al. 2014; Gronke et al. 2016). Herenz et al. (2017) reported a possible observation of such ionized channels seen from the side in the nearby galaxy SBS 0335-052E.

In this Letter, we present rest-frame UV and optical spectroscopy of the lensed galaxy PSZ1-ARC G311.6602-18.4624, which we nickname the Sunburst $\mathrm{Arc}^{1}$. We find that it contains strong evidence for direct escape of Lyman $\alpha$ photons through a perforated optically thick medium. The galaxy was serendipitously discovered in follow-up imaging of its lensing cluster, which was discovered through its Sunyaev-Zel'dovich effect in the Planck data (Planck Collaboration XXIX 2014) and was first reported and described by Dahle et al. (2016). This is to our knowledge the first unambiguous detection of this type of spectral signature, and it bears remarkable resemblance to theoretical predictions of, for instance, Behrens et al. (2014).

\section{Observations and data reduction}

We observed PSZ1-ARC G311.6602-18.4624 through variable cloud cover on UT 24 May 2017, beginning at 03:31, with the Magellan Echellette (MagE) spectrograph (Marshall et al. 2008) on the Magellan-I (Baade) telescope. MagE was configured with the 1 '”0 slit, which delivers a constant spectral resolution as a function of wavelength, $R \simeq 4700\left(\sim 60 \mathrm{~km} \mathrm{~s}^{-1}\right)$ as measured by Gaussian fits to night-sky lines. We performed an alternating sequence of four $2700 \mathrm{~s}$ science frames and $6 \mathrm{~s}$ reference arc lamp calibration frames, with the slit position angle being rotated between exposures to match the parallactic angle at the midpoint of each science frame (see Fig. 2). The seeing varied between $\sim 1{ }^{\prime \prime} 1-11^{\prime \prime} 5$, and the cloud cover increased significantly over the course of the observations (see Fig. 3). We also observed a flux calibrator star, CD-23d (Hamuy et al. 1992, 1994), at three different airmasses bracketing those of the science observations, as well as a second standard, EG131, for correcting telluric absorption lines. The MagE spectra were processed using the MagE pipeline, which is part of the Carnegie Python Distribution $^{2}$, and were fluxed as described in Rigby et al. (2017). Because of the variable cloud cover, we only recovered a relative

\footnotetext{
1 A sunburst being a "direct view of the Sun through rifted clouds".
}

2 Described at http://code.obs. carnegiescience.edu/

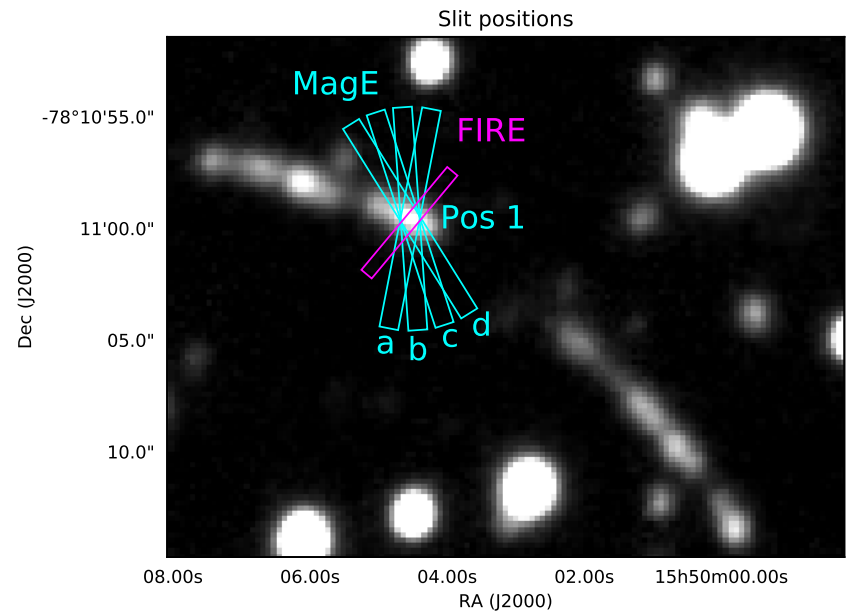

Fig. 2. NTT/EFOSC2 $R$-band image of PSZ1-ARCG311.660218.4624, with MagE (cyan) and FIRE (magenta) slit pointings overlaid. $\mathrm{N}$ is up, $\mathrm{E}$ is left.

flux calibration as a function of wavelength, with no constraint on the absolute flux normalization.

We also observed the arc on UT 30 March 2016, beginning at 09:06 hr, with the Folded-port InfraRed Echelle (FIRE) spectrograph (Simcoe et al. 2013) on the Baade telescope. Observations were performed with the echelle grating and the 0 ".6 slit as a pair of $1800 \mathrm{~s}$ exposures with the target nodded between two positions along the slit, and the slit aligned perpendicular to the arc (see Fig. 2). Conditions were clear throughout the observations, with seeing averaging $\sim 0 \prime \prime 8$. We observed the standard star HIP77712 immediately before the science observations to provide a flux calibration and telluric corrections. The data were reduced using the FireHose v2 data reduction pipeline (Gagné et al. 2015). The final reduced spectrum has a spectral resolution of $R \simeq 6000\left(\sim 50 \mathrm{~km} \mathrm{~s}^{-1}\right)$. The wavelength ranges covered are 3200-8200 $\AA$ for MagE and $0.82-2.49 \mu$ for FIRE. A more comprehensive analysis of these and additional observations is to be presented in a subsequent paper (Rivera-Thorsen et al., in prep.).

We determined the systemic redshift of the arc based on the FIRE spectrum by fitting a single Gaussian profile to each of the four strong emission lines $\mathrm{H} \beta,[\mathrm{O} \mathrm{III}] \lambda \lambda(4959,5007)$, and $\mathrm{H} \alpha$. We then computed the uncertainty-weighted average of the four redshifts to be $z_{\text {neb }}=2.37094 \pm 0.00001$.

\section{Results}

\subsection{Triple-peaked Lyman $\alpha$ emission}

Figure 3 shows cutouts of Ly $\alpha$ from the reduced 2D MagE spectra of the four pointings shown in Fig. 2. The top panel contains the coadded and extracted 1D profile on the same scale. The bright narrow peak at $v \approx 0 \mathrm{~km} \mathrm{~s}^{-1}$ is immediately evident for all of the position angles, and the strongly similar line morphologies point to the flux originating in the same spatial region, at least down to the scale of the seeing. The $1 \mathrm{D}$ profile is a textbook example of the type of line shape we would expect to observe when looking through a clear channel in an otherwise optically thick neutral medium; consisting of a double-peaked profile typical for the Ly $\alpha$ profile emerging through a large number of scatterings with a narrow non-scattered emission peak at line center superimposed onto it, as shown, for example, in Fig. 7 of Behrens et al. (2014). Behrens et al. (2014) did not systematically evaluate their models in a region of parameter space, so 


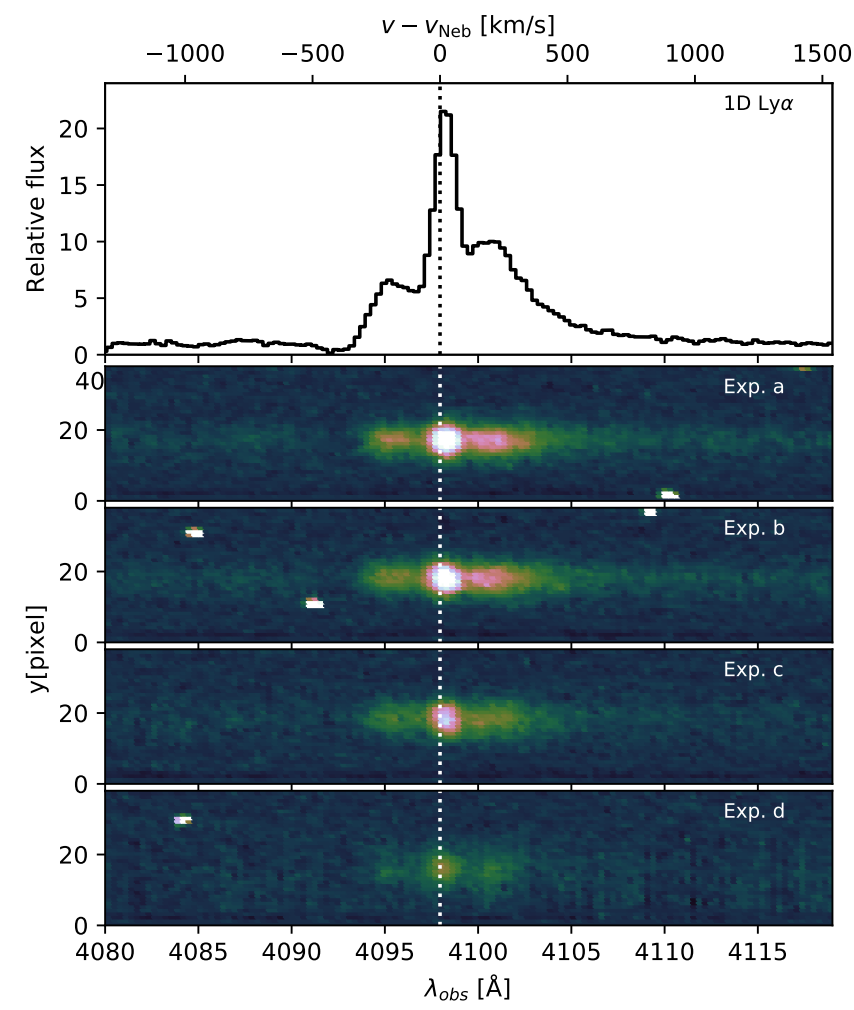

Fig. 3. Top panel: observed Ly $\alpha$ in black. The error spectrum, not shown, is comparable to the line width. Lower panels: two-dimensional Ly $\alpha$ profiles of the four MagE exposures. The color scale is linear, and cut levels are set to enhance detail. The vertical dotted line indicates the best-fit velocity zero-point from rest frame optical nebular transitions.

a quantitative comparison is not possible at the moment, but we plan to model this in Rivera-Thorsen et al. (in prep). It is clear, however, that unlike the typical optically thin density-bounded scenario, this profile also shows strong signs of radiative transfer in at least a modestly thick surrounding neutral medium.

\subsection{Lyman $\alpha$ radiative transfer modeling}

To test whether the observed profile is consistent with the perforated neutral shell scenario, we have attempted to fit the grid of isotropic, expanding shell models presented in Gronke et al. (2015; inspired by similar models by Ahn et al. 2003; Verhamme et al. 2006; Schaerer et al. 2011) to the observed Ly $\alpha$ profile (see Fig. 4). As a prior to the intrinsic profile, we used the width and position of observed $\mathrm{H} \alpha$. We first attempted to fit the full observed Ly $\alpha$ profile to the shell model grid (left panel), but could not even approximately reproduce it, even though shell models are able to reproduce the vast majority of Ly $\alpha$ emission profiles remarkably well (e.g., Gronke 2017). We then fit the central narrow peak to a single Gaussian profile, which was subtracted, and the remaining profile, similar to typical doublepeaked Ly $\alpha$ profiles, was again fitted to the shell model grid (right panel). Unlike the full observed profile, we were able to reproduce the approximated double-peak profile. The fit should not be taken too literally, as the subtraction of the central peak is a coarse approximation, but it does support the hypothesis of a double-peak profile resulting from substantial radiative transfer, with a largely unaltered intrinsic component superimposed upon it.

We then compared the narrow central peak to the shape of $\mathrm{H} \alpha$ to test whether this component is consistent with direct
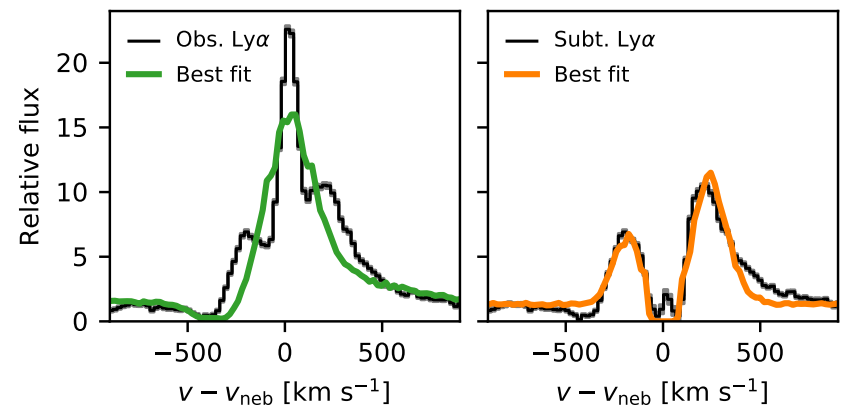

Fig. 4. Best fits of expanding isotropic shell models to the observed data (left) and without the central peak (right).

escape. If no neutral hydrogen is encountered along the LOS, these two profiles will be identical up to a multiplicative constant. Significant amounts of H I, on the other hand, will result in a Ly $\alpha$ line broadened by frequency diffusion. The upper panel in Fig. 5 shows for comparison the normalized Ly $\alpha$ and $\mathrm{H} \alpha$ profiles. $\mathrm{H} \alpha$, observed with FIRE, has been smoothed to match the poorer instrument resolution of MagE and scaled to match the height of $\operatorname{Ly} \alpha$. The profiles of $\operatorname{H} \alpha$ and the central Ly $\alpha$ peak follow each other very well on the red side, as would be expected from the perforated optically thick scenario. Surprisingly, Ly $\alpha$ is narrower than the intrinsic line shape on the blue side, suggesting a weak interaction with neutral gas that absorbs a minor fraction of the light. We interpret this as being due to a residual neutral component in diffuse ionized interstellar or circumgalactic gas along the LOS and note that the low-velocity Si IV feature in Fig. 5 lines up well with the blue edge of the central Ly $\alpha$ peak.

\subsection{Interstellar metal lines}

The lower two panels of Fig. 5 show a selection of metal lines of the neutral and ionized phase. These lines were normalized by fitting a linear function to the local continuum and dividing by the resulting line. We have omitted transitions blueward of rest frame $\operatorname{Ly} \alpha$ due to Ly $\alpha$ forest absorption. The data we show were smoothed by a boxcar kernel of 3 pixels width. Two main components of absorbing gas are evident in the Si IV spectra; one at low velocity $\left(v \sim-100 \mathrm{~km} \mathrm{~s}^{-1}\right)$, and one at intermediate velocity $\left(v \sim-420 \mathrm{~km} \mathrm{~s}^{-1}\right)$. The neutral line profiles are much shallower but follow the general morphology of the ionized medium, reflecting that the majority of ionized gas is likely to be found in the same cloud systems as the neutral gas, on the side facing the central ionizing source.

The neutral lines in the low-velocity component have approximately the same depth, suggesting that the gas is concentrated in optically thick clumps of low velocity-binned covering fraction (see, e.g., Rivera-Thorsen et al. 2017). In contrast, the strong and weak absorption lines behave very differently in the $v \sim-420 \mathrm{~km} \mathrm{~s}^{-1}$ component, with the weak lines being either non-detections or extremely shallow. A quantitative analysis of this is to be made in our follow-up paper, but this shows that at least at the blue end, the neutral gas is optically thin, at least to the weaker transitions, and thus might cover the background fully. At this velocity, it has little impact on the line shape of Ly $\alpha$, but it may still substantially affect LyC escape.

All the ions shown in the middle panel of Fig. 5 have finestructure split ground levels, and thus some amount of fluorescent emission is expected. As argued by Jaskot \& Oey (2014), a comparison of resonant absorption depth and fluorescent emission strength might indicate neutral gas within the spectrograph 


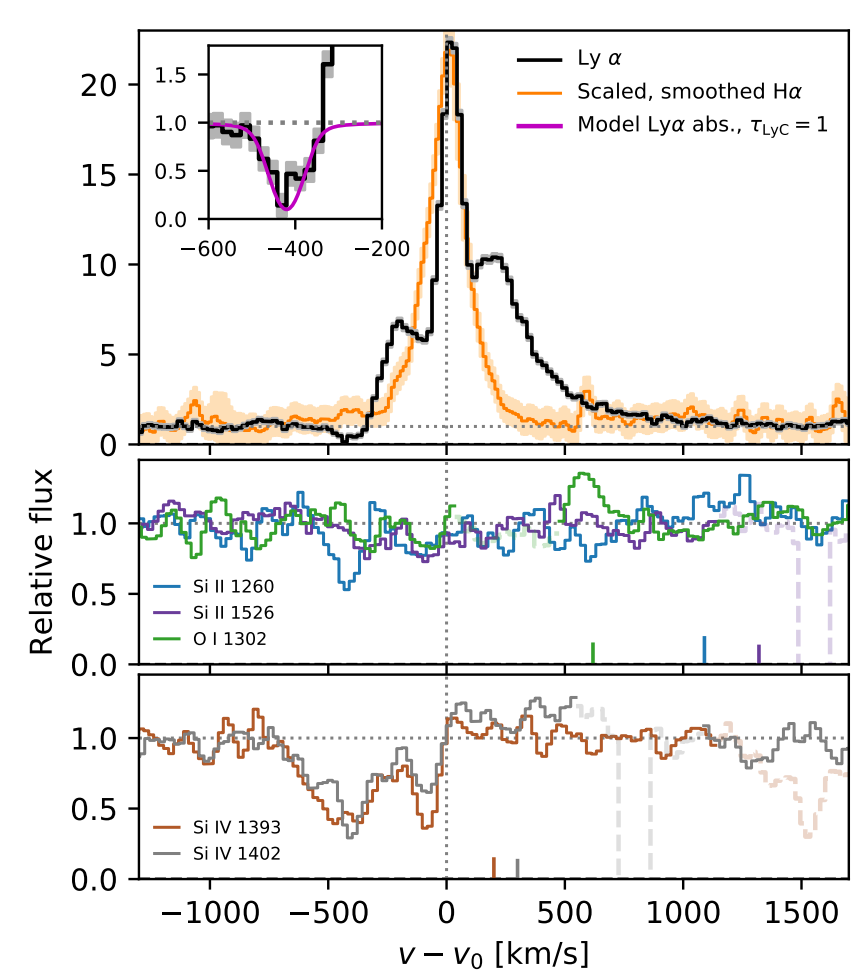

Fig. 5. Continuum-normalized line profiles. Upper panel: Lyman $\alpha$ and smoothed, scaled $\mathrm{H} \alpha$ (see text for details). Inset: zoom-in on Ly $\alpha$, with the theoretical absorption profile for $\tau_{\mathrm{LyC}}=1$ superimposed. Middle panel: selected low-ionization metal absorption. Colored markers show the approximate expected position of fluorescent emission lines, color coded by their corresponding resonant line, with lengths indicating the average error spectrum in a velocity range of $\pm 1500 \mathrm{~km} \mathrm{~s}^{-1}$ around the line. Lower panel: Si IV. Colored markers show the average errors, but are placed at arbitrary wavelengths. Metal lines are smoothed by 3 px. Gray dotted lines denote continuum level (horizontal) and velocity zero point (vertical). Pale dashed lines show regions masked out due to contamination.

aperture, but off the LOS. We have shown the approximate expected centroids of fluorescent emission in Fig. 5. Despite the very shallow absorption, at least some of these lines show significant fluorescent emission, suggesting the presence of significant amounts of off-LOS neutral gas, in agreement with what is expected from the radiative transfer effects seen in $\operatorname{Ly} \alpha$.

\subsection{Conditions for Lyman continuum escape}

The presence of empty or highly ionized LOS makes the Sunburst Arc a prime candidate for spatially resolved observation of LyC. Some uncertainty arises from the component at $v \sim$ $-420 \mathrm{~km} \mathrm{~s}^{-1}$. Figure 5 shows that the Si II lines have a strongly differing depth, implying that this component is optically thin at least in $\lambda 1526$ and possibly also in $\lambda 1260$, in which case the neutral gas component might be covering the entire background source. Because of the high velocity, this has little impact on Ly $\alpha$ escape, but $\mathrm{LyC}$ is sensitive to neutral gas at any velocity and might be blocked by this system. A full analysis of this is planned for a follow-up paper, but as a first test, we modeled the theoretical Ly $\alpha$ absorption profile arising from a gas component at $v=-420 \mathrm{~km} \mathrm{~s}^{-1}$ with $N_{\mathrm{HI}}=10^{17.2} \mathrm{~cm}^{-2}$ (corresponding to $\left.\tau_{\mathrm{LyC}}=1, f_{\mathrm{esc}}(\mathrm{LyC}) \sim 0.4\right)$ ) and $T=10^{4} \mathrm{~K}$. The resulting profile, convolved with the MagE instrument profile, is shown in magenta in the inset of Fig. 5. The theoretical line is too strong to be consistent with the observed feature. We tentatively conclude that the neutral component at $v \sim-420 \mathrm{~km} \mathrm{~s}^{-1}$ either has too low column density or covering fraction to effectively block LyC escape. An HST mid-cycle proposal to image the arc in rest-frame LyC has recently been accepted.

\section{Summary}

We have presented Magellan/MagE and Magellan/FIRE spectra of the extremely bright strongly lensed galaxy PSZ1ARC G311.6602-18.4624, which we nickname the Sunburst Arc. By comparing the 2D spectra of four different position angles, we find that it is unlikely that any contamination from nearby objects is present. Based on strong Balmer- and [O III] emission lines, we have improved the precision of previous redshift estimates. From this, we found that the Ly $\alpha$ line shape in the combined MagE spectrum closely matches theoretical predictions for direct Ly $\alpha$ escape through a perforated neutral medium. By comparing to the $\mathrm{H} \alpha$ emission feature, we found that this narrow central peak has most likely undergone no other radiative transfer than very slight absorption, probably by a residual neutral fraction of $\mathrm{HI}$ in the ionized medium along the open LOS. The observed line profile cannot be modeled by isotropic expanding shell models, but a reasonable fit can be obtained by removing the central peak. A medium-velocity neutral gas component at $v \sim-420 \mathrm{~km} \mathrm{~s}^{-1}$ causes some uncertainty as to whether the LOS seen in Ly $\alpha$ would also be transparent in LyC, but the absorption feature it leaves in the Ly $\alpha$ continuum suggests that it is either optically thin to LyC or does not cover the background completely.

Acknowledgements. We thank the anonymous referee for thorough and constructive suggestions, and E. C. Herenz for useful comments and ideas. E.R.-T. thanks Stockholm University for their kind hospitality. E.R.-T. and H.D. acknowledge support from the Research Council of Norway.

\section{References}

Ahn, S.-H., Lee, H.-W., \& Lee, H. M. 2003, MNRAS, 340, 863 Behrens, C., Dijkstra, M., \& Niemeyer, J. C. 2014, A\&A, 563, A77 Bouwens, R. J., Illingworth, G. D., Oesch, P. A., et al. 2012, ApJ, 752, L5 Chisholm, J., Orlitová, I., Schaerer, D., et al. 2017, A\&A, 605, A67 Conselice, C. J., Gallagher, J. S., Calzetti, D., Homeier, N., \& Kinney, A. 2000, AJ, 119, 79

Dahle, H., Aghanim, N., Guennou, L., et al. 2016, A\&A, 590, L4

Dijkstra, M. 2014, PASA, 31, 40

Duval, F., Schaerer, D., Östlin, G., \& Laursen, P. 2014, A\&A, 562, A52 Faisst, A. L. 2016, ApJ, 829, 99

Gagné, J., Lambrides, E., Faherty, J. K., \& Simcoe, R. 2015, Firehose v2.0 DOI: $10.5281 /$ zenodo. 18775

Gronke, M. 2017, A\&A, in press, DOI: 10.1051/0004-6361/201731791

Gronke, M., Bull, P., \& Dijkstra, M. 2015, ApJ, 812, 123

Gronke, M., Dijkstra, M., McCourt, M., \& Oh, S. P. 2016, ApJ, 833, L26

Hamuy, M., Walker, A. R., Suntzeff, N. B., et al. 1992, PASP, 104, 533

Hamuy, M., Suntzeff, N. B., Heathcote, S. R., et al. 1994, PASP, 106, 566

Hansen, M., \& Oh, S. P. 2006, MNRAS, 367, 979

Heckman, T. M., Borthakur, S., Overzier, R., et al. 2011, ApJ, 730, 5

Herenz, E. C., Hayes, M., Papaderos, P., et al. 2017, A\&A, 606, L11

Jaskot, A. E., \& Oey, M. S. 2013, ApJ, 766, 91

Jaskot, A. E., \& Oey, M. S. 2014, ApJ, 791, L19

Marshall, J. L., Burles, S., Thompson, I. B., et al. 2008, in Ground-based and Airborne Instrumentation for Astronomy II, Proc. SPIE, 7014, 701454

Osterbrock, D. E., \& Ferland, G. J. 2006, Astrophysics of gaseous nebulae and active galactic nuclei (CA: University Science Books)

Planck Collaboration XXIX. 2014, A\&A, 571, A29

Rigby, J. R., Bayliss, M. B., Sharon, K., et al. 2017, ArXiv e-prints [arXiv: 1710.07294]

Rivera-Thorsen, T. E., Östlin, G., Hayes, M., \& Puschnig, J. 2017, ApJ, 837, 29 Schaerer, D., Hayes, M., Verhamme, A., \& Teyssier, R. 2011, A\&A, 531, A12 Simcoe, R. A., Burgasser, A. J., Schechter, P. L., et al. 2013, PASP, 125, 270 Verhamme, A., Schaerer, D., \& Maselli, A. 2006, A\&A, 460, 397 Verhamme, A., Orlitová, I., Schaerer, D., \& Hayes, M. 2015, A\&A, 578, A7 Zackrisson, E., Inoue, A. K., \& Jensen, H. 2013, ApJ, 777, 39 